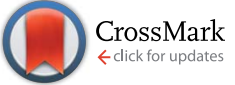

Cite this: RSC Adv., 2017, 7, 17353

Received 24th January 2017

Accepted 15th March 2017

DOI: 10.1039/c7ra01091e

rsc.li/rsc-advances

\title{
Synthesis and water sorption properties of a series of exfoliated graphene/MIL-100(Fe) composites $\dagger$
}

\author{
S. A. M. Said, ${ }^{\star a b}$ N. U. Qadir, ${ }^{a}$ R. B. Mansour, ${ }^{\text {bc }}$ K. Mezghani ${ }^{b c}$ and H. M. Irshad ${ }^{c}$ \\ A novel composite composed of exfoliated graphene nanoplatelets (Ex-GNPs) incorporated in a MIL- \\ 100(Fe) framework has been synthesized using an in situ molecular-level interaction approach. The \\ resulting Ex-GNP/MIL-100(Fe) composite, with varying Ex-GNP contents, has been characterized for \\ microstructure, degree of crystallinity, thermal stability, water sorption behavior, and hydrothermal cyclic \\ stability
}

\section{Introduction}

Metal Organic Frameworks (MOFs) have attracted immense attention owing to their high specific surface area and pore volume apart from the unprecedented versatility in metal-clusterlinker combination reported for these materials. ${ }^{\mathbf{1 - 1 0}}$ However, in the context of water adsorption, very few amongst the wide variety of these novel materials have been observed to retain structural stability in aqueous environments. MIL-100(Fe) and MIL-101(Cr) represent two such water-stable MOFs which have not only been characterized by high water vapour uptakes of 0.65 and $1.4 \mathrm{~g} \mathrm{~g}^{-1}$ respectively, but also excellent stability under repeated cyclic adsorption-desorption in humid environments up to $140{ }^{\circ} \mathrm{C}^{\mathbf{4 , 1 0}}$ However, owing to the presence of an organic portion which is present in significantly larger proportion than the metal ion centers, the thermal conductivity exhibited by both these waterstable MOFs is still insufficient in the context of use as an adsorbent in a chiller application. ${ }^{\mathbf{1 1} 12}$

Multi-walled carbon nanotubes (MWCNTs) have been recently utilized as supports for the nucleation and growth of MIL-100(Fe) crystals in order to improve the heat transfer characteristics of the MWCNT/MIL-100(Fe) composite. ${ }^{13}$ However, the inherent tendency of MWCNTs to agglomerate inside aqueous suspensions has been observed to lead to a discontinuous trend of variation in water sorption characteristics of the composite material with increasing content of MWCNTs inside MIL-100(Fe). Unlike MWCNTs, graphene nanoplatelets (GNPs) incorporate a plate-like morphology which not only offer a higher surface area for the growth of MOF

${ }^{a}$ Center of Research Excellence in Renewable Energy, King Fahd University of Petroleum and Minerals (KFUPM), Dhahran 31261, Kingdom of Saudi Arabia

${ }^{b}$ KACST-TIC Center of Carbon Capture and Sequestration, KFUPM, Dhahran 31261, Kingdom of Saudi Arabia. E-mail: samsaid@kfupm.edu.sa

${ }^{c}$ Department of Mechanical Engineering, KFUPM, Dhahran 31261, Kingdom of Saudi Arabia

$\dagger$ Electronic supplementary information (ESI) available. See DOI: 10.1039/c7ra01091e crystals, but also exhibit a much lesser tendency towards aggregation inside an aqueous medium. In this work, we have proposed GNPs as a potential replacement of MWCNTs as additives inside the MIL-100(Fe) matrix with the same objective of improving the thermal characteristics of the base MOF. For achieving a robust chemical interface between the supports and the crystals, a prior acid-treatment of GNPs resulting in the formation of exfoliated GNPs (Ex-GNPs) has been conducted. The water sorption kinetics of the resulting Ex-GNP/MIL-100(Fe) composite have been evaluated in the context of its potential use in an adsorption chiller application.

The synthesis and activation of MIL-100(Fe) was carried out according to a previously reported procedure (see ESI, Section $\mathrm{S} 3 \dagger) .{ }^{16}$ For the Ex-GNP/MIL-100(Fe) composites, the first step of synthesis involves the attachment of carboxyl $(\mathrm{O}=\mathrm{C}-\mathrm{OH}$ and $\mathrm{C}-$ $\mathrm{OH})$ moieties on the surface of graphene nanoplatelets (GNPs) using acid-functionalization resulting in the formation of exfoliated graphene nanoplatelets (Ex-GNPs). The attachment of these carboxyl groups on the surface of GNPs was chemically proven using Fourier Transform Infrared Spectroscopy (FT-IR) (ESI, Fig. S3†). These carboxyl functionalities are later converted into $\mathrm{C}-\mathrm{O}-\mathrm{Fe}$ linkages upon sonication-assisted interaction with $\mathrm{Fe}^{3+}$ ions in a common solvent (Scheme 1c). The subsequent hydrothermal synthesis in the presence of 1,3,5benzenetricarboxylic acid $\left(\mathrm{H}_{3}\right.$-BTC) finally results in MIL$100(\mathrm{Fe})$ crystals which are either wrapped by the covalently attached Ex-GNPs in order to attain the minimum energy configuration (Scheme 1d), or are embedded on the neighbouring Ex-GNPs if the added content of Ex-GNPs is sufficiently large. The synthesized Ex-GNP/MIL-100(Fe) composites are referred to as MIL-G1, MIL-G2 and MIL-G3 based upon the added content of Ex-GNPs in the MIL-100(Fe) precursor during synthesis (70,180 and $300 \mathrm{mg}$ respectively). The final content of Ex-GNPs in Ex-GNP/MIL-100(Fe) composites was evaluated using both elemental analysis (EA) and thermogravimetric analysis (TGA). Based on EA data, the contents of Ex-GNPs were found to be 1.88, 5.56 and $15.10 \mathrm{wt} \%$ for MIL-G1-3, respectively 
(a)

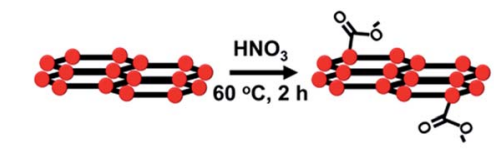

(b)

$$
n \mathrm{Fe}\left(\mathrm{NO}_{3}\right)_{3} \cdot 9 \mathrm{H}_{2} \mathrm{O} \underset{\text { water }}{\stackrel{\text { Deionized }}{\longrightarrow}} n \mathrm{Fe}^{3+}+3 n \mathrm{NO}_{3}{ }^{-}+9 n \mathrm{H}_{2} \mathrm{O}
$$

(c)

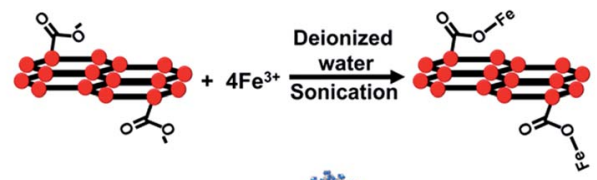

(d)

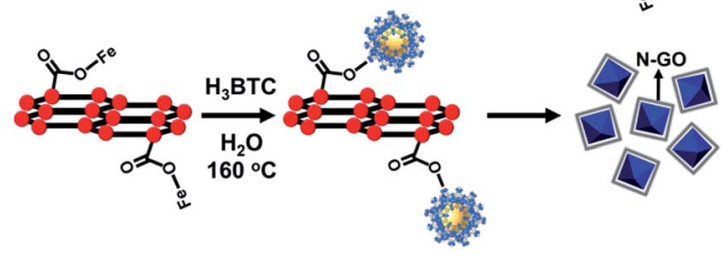

Scheme 1 (a) Chemical functionalization of a graphene nanoplatelet resulting in the attachment of negatively-charged carboxyl groups (Ex-GNPs), (b) dissociation of precursor salt in aqueous medium, (c) sonication-assisted interaction of carboxyl groups on Ex-GNPs with $\mathrm{Fe}^{3+}$ ions in aqueous medium, (d) hydrothermal synthesis resulting in Ex-GNP/MIL-100(Fe) composites in which the Ex-GNPs are wrapped around the MIL-100(Fe) crystals.

(ESI, Section S5 $†$ ). The corresponding values measured using TGA data were observed to be $6.54,12.93$ and $16.16 \mathrm{wt} \%$, respectively (ESI, Table S1 $\dagger$ ). For this study, the values obtained using TGA data have been preferred since the accuracy of EA data in the determination of Ex-GNP content has been observed to rely upon the efficiency of the activation procedure. ${ }^{17}$ However, the chemical formulation of each of the activated MOF materials has been determined using the percentages of $\mathrm{C}$, $\mathrm{H}$ and $\mathrm{N}$ in the framework obtained using EA. It has been observed that the MIL-G3 framework loses virtually all of its water of crystallization upon activation and thus transforms from a hydrated to a completely dehydrated structure due to the

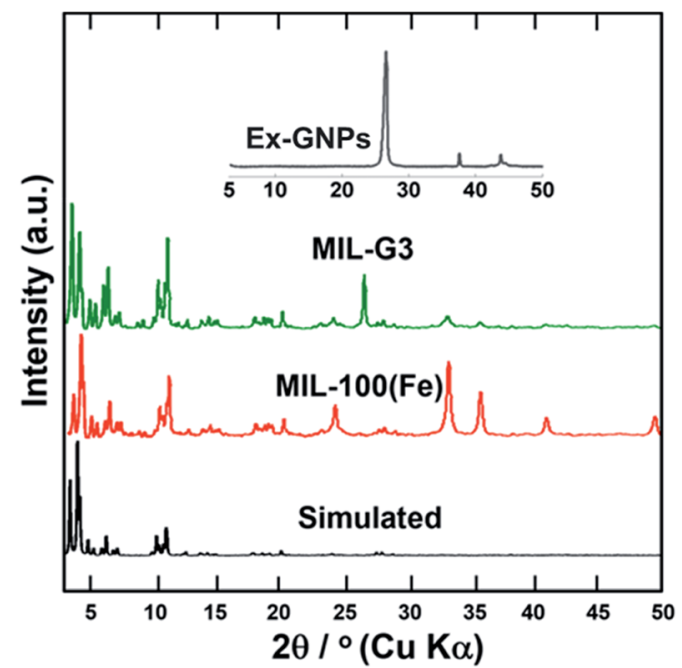

Fig. 1 PXRD patterns of MIL-100(Fe) and Ex-GNP/MIL-100(Fe) composites.
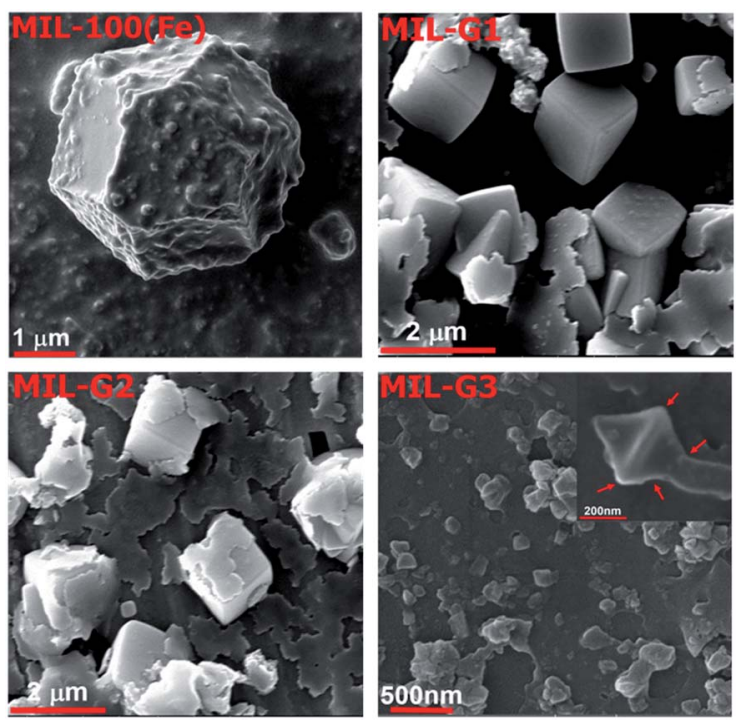

Fig. 2 SEM micrographs of MIL-100(Fe) and Ex-GNP/MIL-100(Fe) composites (red arrows in the inset of MIL-G3 show an isolated MIL100(Fe) crystal embedded on a neighbouring Ex-GNP).

incorporation of a substantially large amount of Ex-GNPs inside the MIL-100(Fe) framework (ESI, Section S5 $\dagger$ ).

Fig. 1 shows a comparison of the powder X-ray diffraction (PXRD) patterns of MIL-100(Fe) and MIL-G3. It can be seen that the PXRD pattern of MIL-100(Fe) is in good agreement with the simulated pattern from the single crystal structure. It is observed that the addition of Ex-GNPs does not result in any noticeable peak shifts in the PXRD patterns of MIL-G1-3 (ESI, Fig. S2 $\dagger$ ). Hence, the introduction of graphene up to $16 \mathrm{wt} \%$ does not disrupt the intrinsic crystal structure of MIL-100(Fe). However, an extra peak at $2 \theta=26.06^{\circ}$ has been observed in the PXRD profiles of MIL-G1-3 owing to the reflections from the (001) planes of graphitic carbon inside Ex-GNPs (ESI, Section S9†). The intensity of these reflections has been observed to be much greater in ExGNPs as compared to the pristine GNPs owing to the higher interaction volume available to incident X-rays due to the exfoliation of GNPs upon acid-treatment (ESI, Section S4†). From the nitrogen adsorption-desorption isotherms measured at $77 \mathrm{~K}$, the Brunauer-Emmett-Teller (BET) surface area for MIL-100(Fe) has been evaluated to be $1360 \mathrm{~m}^{2} \mathrm{~g}^{-1}$ which is in good agreement with literature (ESI, Fig. S5†). ${ }^{16}$ The highest specific surface area and pore volume are exhibited by MIL-G3, while both MIL-G1 and
(A)

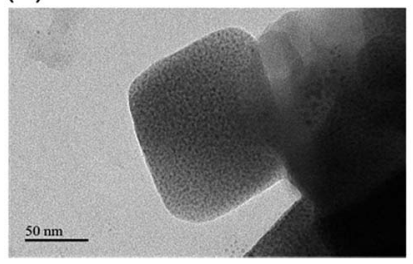

(B)

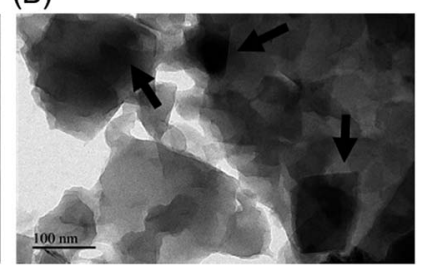

Fig. 3 TEM micrographs of (A) MIL-100(Fe) and (B) MIL-G2 (black arrows point at MIL-100(Fe) crystals embedded on the neighbouring Ex-GNPs). 
(A)

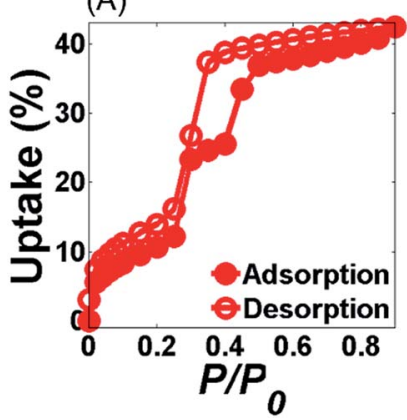

(B)

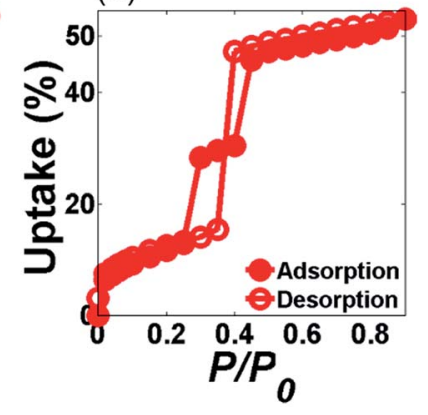

Fig. 4 Water adsorption-desorption isotherms of (A) MIL-100(Fe) and (B) MIL-G3 at $298 \mathrm{~K}$.

Table 1 Water sorption properties of MIL-100(Fe) and Ex-GNP/MIL100(Fe) composites

\begin{tabular}{lll}
\hline Sample ID & $\begin{array}{l}\text { Water uptake } \\
(\%)\end{array}$ & $\begin{array}{l}\text { Uptake loss b/w } \\
\text { first and last cycle }(\%)\end{array}$ \\
\hline MIL-100(Fe) & 42.45 & 48.20 \\
Ex-GNPs & 2.653 & N.A. \\
MIL-G1 & 40.62 & 27.57 \\
MIL-G2 & 33.92 & 48.24 \\
MIL-G3 & 53.05 & 29.00
\end{tabular}

MIL-G2 have been noticed to show lower surface areas and pore volumes than MIL-100(Fe) (ESI, Table S2 †).

The repulsive forces between the $\mathrm{COOH}^{-}$groups helps to overcome the inherent van der Waals attraction between the neighbouring platelets, thus facilitating their dispersion in an aqueous medium (ESI, Section S11†). The morphologies of MIL-
100(Fe) and Ex-GNP/MIL-100(Fe) composites as observed by Scanning Electron Microscopy (SEM) are shown in Fig. 2. The incorporation of Ex-GNPs is observed to preserve the intrinsic octahedral morphology of MIL-100(Fe) crystals. It is observed that the crystals are predominantly wrapped by the surrounding platelets for the case of MIL-G1 and MIL-G2 (Scheme 1d); however, since the size of MIL-100(Fe) crystals becomes sufficiently small in case of MIL-G3 due to the higher added content of Ex-GNPs, they are primarily observed to be embedded on the surrounding Ex-GNPs as apparent in the inset shown in the SEM image of MIL-G3. ${ }^{14}$ Fig. 3 shows the Transmission Electron Microscopy (TEM) micrographs of MIL-100(Fe) and MIL-G3. The TEM micrograph of MIL-G3 shows a number of MIL-100(Fe) crystals (black arrows) embedded on the neighbouring Ex-GNPs owing to the sufficiently smaller crystal size in accordance with a large added content of Ex-GNPs. In contrast, the micrograph of MIL-G1 clearly shows a dark-coloured corner of a single platelet reflected on the rear side of an isolated MIL-100(Fe) crystal which is a confirmation of the partial wrapping of the crystal by Ex-GNPs in accordance with the proposed scheme of synthesis (ESI, Fig. S13†). The Fourier Transform Infrared (FT-IR) spectrum measured for MIL-100(Fe) is in good agreement with the already reported literature for a similar method of synthesis (ESI, Fig. S16 $\dagger$ ). ${ }^{18}$ However, for MIL-G1-3, a relatively wider vibration band noted around $3445 \mathrm{~cm}^{-1}$ which is assignable to the $\mathrm{O}-\mathrm{H}$ stretch of the carboxylic acid groups attached on the surface of ExGNPs (ESI, Fig. S3 $\dagger$ ). A weak signal observed in the spectra of MILG2 and MIL-G3 around $500-515 \mathrm{~cm}^{-1}$, which is absent in the spectrum of MIL-100(Fe), confirms the existence of the proposed $\mathrm{C}-\mathrm{O}-\mathrm{Fe}$ linkage between the MIL-100(Fe) crystals and the ExGNPs in the Ex-GNP/MIL-100(Fe) composites (ESI, Fig. S17†). ${ }^{19}$ This was further confirmed using the X-ray photoelectron spectroscopy (XPS) analysis conducted on MIL-100(Fe) and MIL-G2
(A)
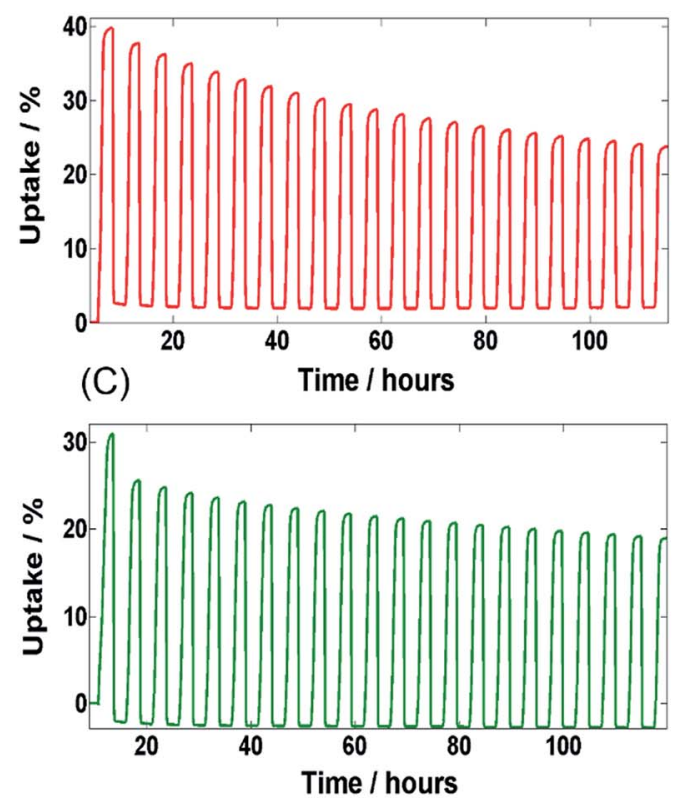

(B)
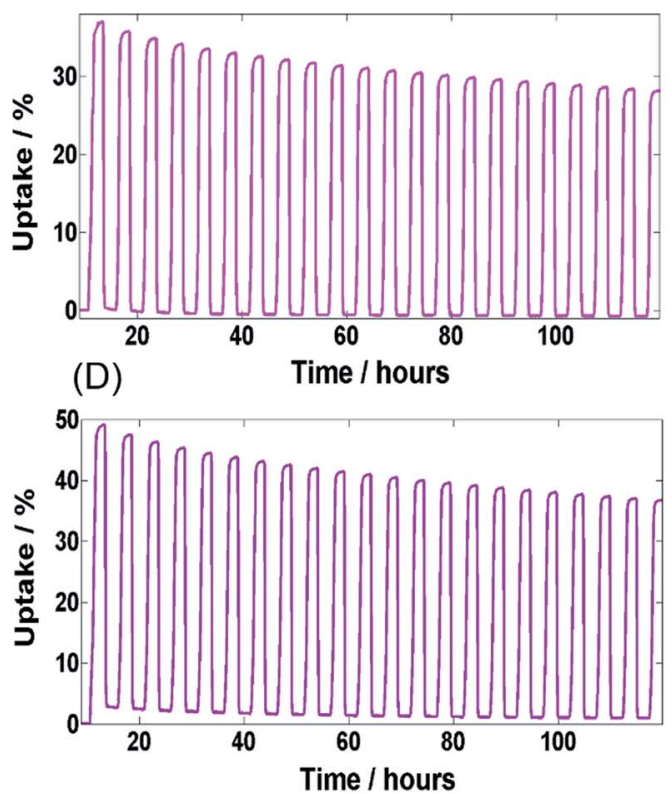

Fig. 5 Cyclic adsorption-desorption profiles for 21 cycles - (A) MIL-100(Fe), (B) MIL-G1, (C) MIL-G2 and (D) MIL-G3. 
(ESI, Table S3 $\dagger$ ). ${ }^{\mathbf{1 3}}$ The thermogravimetric analysis (TGA) profile of MIL-100(Fe) exhibits three major weight losses between 30 and $700{ }^{\circ} \mathrm{C},{ }^{16}$ whereas an additional weight loss is observed between 350 and $700{ }^{\circ} \mathrm{C}$ in case of MIL-G1-3 owing to the oxidation of graphene (ESI, Fig. S19 $\dagger$ ). The residue recovered after the TGA was identified using PXRD to be predominantly $\alpha-\mathrm{Fe}_{2} \mathrm{O}_{3}$ (ESI, Fig. S20 $\dagger) .{ }^{\mathbf{1 3}}$ The TGA profiles of MIL-G1-3 do not reveal any noticeable differences between 40 and $140{ }^{\circ} \mathrm{C}$, which marks the useful working temperature range of a typical adsorption chiller. ${ }^{10}$

Fig. 4 shows the water adsorption-desorption isotherms of MIL-100(Fe) and MIL-G3 measured at $298 \mathrm{~K}$. The water sorption kinetics observed for MIL-100(Fe) are in agreement with the already published literature. ${ }^{\mathbf{1} 15}$ For MIL-G1-3, it can be noticed that the adsorption branch remains more or less unaffected by the addition of Ex-GNPs up to $16 \mathrm{wt} \%$ (ESI, Section $\mathrm{S} 17 \dagger$ ). However, the desorption profiles of MIL-G1 and MIL-G2 show an abrupt loss in uptake for $0.4<P / P_{0}<0.5$, which shifts to $0.3<P / P_{0}$ $<0.4$ for the case of MIL-G3. This is attributable to the increased hydrophobicity of the framework due to the incorporation of graphene which accelerates the desorption of water molecules when a critical value of $P / P_{0}$ is reached..$^{20}$ The highest uptake has been recorded for MIL-G3, which is observed to be $22 \%$ higher than that measured for MIL-100(Fe) (Table 1). For MIL-G1-3, it is noticed that the desorption branch intersects the adsorption branch at a critical value of $P / P_{0}$. This is also attributable to the presence of graphene since the water sorption isotherm of ExGNPs has also been observed to show the same behaviour (ESI, Fig. S20 $\dagger$ ). It can be seen that MIL-G3 not only results in the lowest value of $P / P_{0}$ corresponding to half of the maximum uptake amongst the three composites, but also a degree of hysteresis between the adsorption and desorption phases which is almost $120 \%$ lower as compared to the value measured for MIL-100(Fe) (ESI, Table S4 $\dagger$ ). Since the existence of a minimum hysteresis is considered to be a major prerequisite for any adsorbent material designed for a chiller application, ${ }^{\mathbf{1 0}}$ MIL-G3 thus presents an attractive alternative to the more conventional silica-gel- and zeolite-based adsorbents in this context. The cyclic water adsorption/desorption test results based on 21 consecutive cycles for MIL-100(Fe) and Ex-GNP/MIL-100(Fe) composites are shown in Fig. 5 . The best cycling performance is exhibited by MIL-G1 which results in an uptake loss of only $28 \mathrm{wt} \%$ measured between the first and the last cycles. It is observed that MIL-G3 results in an almost 50\% lower uptake loss between the first and the last cycles as compared to MIL-100(Fe) (Table 1). SEM and PXRD analyses conducted on the cycled samples indicate that each retains its characteristic octahedral morphology following the cyclic adsorption-desorption test (ESI, Section S20 $\dagger$ ).

In conclusion, the molecular-level approach proposed for the synthesis of Ex-GNP/MIL-100(Fe) composites has been observed to preserve the characteristic lattice structure and chemistry of the MIL-100(Fe) framework for an addition of Ex-GNPs up to 16 wt $\%$. The composite containing 16 wt $\%$ Ex-GNPs has been evaluated to result in an almost $10 \%$ higher specific surface area, a $22 \%$ higher water vapour uptake at $298 \mathrm{~K}$, a $50 \%$ higher hydrothermal cyclic stability, and a $120 \%$ lower degree of hysteresis between the adsorption and the desorption phases than the corresponding values measured for unmodified MIL-
100(Fe). The proposed Ex-GNP/MIL-100(Fe) composite thus presents an attractive alternative to the more conventional silica gel- and zeolite-based adsorbents for use in commercial-scale adsorption chillers using water as refrigerant.

\section{Acknowledgements}

The authors are grateful to the technical support granted by the Center of Research Excellence in Renewable Energy, and the Center of Carbon Capture and Sequestration, King Fahd University of Petroleum and Minerals, Dhahran, Saudi Arabia, in all phases of preparation of the manuscript.

\section{Notes and references}

1 F. Jeremias, A. Khutia, S. K. Henninger and C. Janiak, J. Mater. Chem., 2012, 22, 10148.

2 F. Jeremias, D. Fröhlich, C. Janiak and S. K. Henninger, New J. Chem., 2014, 38, 1846.

3 S. K. Henninger, H. A. Habib and C. Janiak, J. Am. Chem. Soc., 2009, 131, 2776.

4 G. Akiyama, R. Matsuda and S. Kitagawa, Chem. Lett., 2010, 39, 360 .

5 J. Canivet, A. Fateeva, Y. Guo, B. Coasne and D. Farrusseng, Chem. Soc. Rev., 2014, 43, 5594.

6 C. Janiak and S. K. Henninger, Chimia, 2013, 67, 419.

7 J. Ehrenmann, S. K. Henninger and C. Janiak, Eur. J. Inorg. Chem., 2011, 2011, 471.

8 S. K. Henninger, G. Munz, K.-F. Ratzsch and P. Schossig, Renewable Energy, 2011, 36, 3043.

9 F. Jeremias, S. K. Henninger and C. Janiak, Chem. Commun., 2012, 48, 9708.

10 S. K. Henninger, F. Jeremias, H. Kummer and C. Janiak, Eur. J. Inorg. Chem., 2012, 2012, 2625.

11 A. Rezk, R. Al-Dadah, S. Mahmoud and A. Elsayed, Int. J. Heat Mass Transfer, 2012, 55, 7366.

12 M. F. de Lange, B. L. van Velzen, C. P. Ottevanger, K. J. F. M. Verouden, L.-C. Lin, T. J. H. Vlugt, J. Gascon and F. Kapteijn, Langmuir, 2015, 31, 12783.

13 N. U. Qadir, S. A. M. Said, R. B. Mansour, K. Mezghani and A. U. Hamid, Dalton Trans., 2016, 45, 15621.

14 Z. Zhao, S. Wang, R. Liang, Z. Li, Z. Shid and G. Chen, J. Mater. Chem. A, 2014, 2, 13509.

15 P. Küsgens, M. Rose, I. Senkovska, H. Fröde, A. Henschel, S. Siegle and S. Kaskel, Microporous Mesoporous Mater., 2009, 120, 325.

16 Y.-K. Seo, J. W. Yoon, J. S. Lee, U.-H. Lee, Y. K. Hwang, C.-H. Jun, P. Horcajada, C. Serre and J.-S. Chang, Microporous Mesoporous Mater., 2012, 157, 137.

17 A. Schaate, P. Roy, A. Godt, J. Lippke, F. Waltz, M. Wiebcke and P. Behrens, Chem.-Eur. J., 2011, 17, 6643.

18 R. Liang, R. Chen, F. Jing, N. Qin and L. Wu, Dalton Trans., 2015, 44, 18227.

19 M. G. I. Galinato, C. M. Whaley and N. Lehnert, Inorg. Chem., 2010, 49, 3201.

20 J. Yan, Y. Yu, C. Ma, J. Xiao, Q. Xia, Y. Li and Z. Li, Appl. Therm. Eng., 2015, 84, 118. 